\title{
Evaluation of contrast visual acuity in patients with retinitis pigmentosa
}

This article was published in the following Dove Press journal:

Clinical Ophthalmology

10 October 2011

Number of times this article has been viewed

\author{
Kazumi Oomachi ${ }^{1}$ \\ Kazuha Ogata ${ }^{2}$ \\ Takeshi Sugawara ${ }^{2}$ \\ Akira Hagiwara ${ }^{2}$ \\ Akira Hata' \\ Shuichi Yamamoto ${ }^{2}$ \\ 'Department of Public Health; \\ ${ }^{2}$ Department of Ophthalmology, \\ Chiba University Graduate School \\ of Medicine, Chiba, Japan
}

Correspondence: Akira Hata Department of Public Health, Chiba University Graduate School of Medicine, I-8-I Inohana, Chiba 260-8670, Japan Tel +8I 432262067

Fax +8I 432262070

Email ahata@faculty.chiba-u.jp
Background: The purpose of this study was to determine visual acuity at different contrast levels under photopic and mesopic conditions in patients with retinitis pigmentosa.

Methods: Sixty eyes of 31 normal controls, 92 eyes of 52 patients with retinitis pigmentosa without other ocular disorders (RP-1 group), and 20 eyes of 14 patients with retinitis pigmentosa with cataracts and without other ocular disorders (RP-2 group) were studied. Conventional visual acuity was measured using a conventional Landolt ring chart with $100 \%$ contrast and luminance of $150 \mathrm{~cd} / \mathrm{m}^{2}$. All of the patients with retinitis pigmentosa had a decimal visual acuity better than 1.0. Contrast visual acuity was measured with the same Landolt ring chart with contrasts of $100 \%$ and $10 \%$ and under photopic $\left(200 \mathrm{~cd} / \mathrm{m}^{2}\right)$ and mesopic $\left(10 \mathrm{~cd} / \mathrm{m}^{2}\right)$ conditions. Decimal visual acuities were converted to $\log$ MAR units for the analyses.

Results: The $100 \%$ contrast visual acuity and the $10 \%$ contrast visual acuity determined under both photopic and mesopic conditions were significantly poorer in both the RP-1 and RP-2 groups than in the controls. The differences between the conventional visual acuity and the $100 \%$ contrast visual acuity were significantly greater in the RP-1 and RP-2 groups than in the controls under both photopic and mesopic conditions. The differences between the $100 \%$ contrast visual acuity and the $10 \%$ contrast visual acuity were not significant among the three groups under photopic and mesopic conditions.

Conclusion: Contrast visual acuities were greatly reduced in patients with retinitis pigmentosa with relatively well preserved conventional visual acuity, and the contrast visual acuity was largely influenced by ambient light levels in patients with retinitis pigmentosa. Although a longitudinal study for confirmation has to be performed, our findings indicate that contrast visual acuity is a better test to follow changes in visual function in patients with retinitis pigmentosa.

Keywords: retinitis pigmentosa, contrast visual acuity, photopic vision, mesopic vision

\section{Introduction}

Retinitis pigmentosa is a collection of inherited retinal disorders characterized by a progressive increase in night blindness and visual field loss. The rod and cone photoreceptors and retinal pigment epithelium are the main structures affected. The number of genes, mutations of which cause retinitis pigmentosa, has greatly increased, but the phenotypes of these patients vary considerably. Even a mutation in one gene can lead to very different clinical phenotypes. Thus, it is difficult to predict the degree of progression and the final visual acuity in individual patients by genotyping. ${ }^{1}$

The two most common tests used in the clinic to follow the progression of retinitis pigmentosa are best corrected visual acuity, measured with optotypes of approximately $100 \%$ contrast, and visual fields. However, these examinations are generally not 
sensitive enough to evaluate the degree of progression of retinitis pigmentosa. Several studies have used contrast sensitivity measurements to evaluate patients with retinitis pigmentosa, and the results have shown that the contrast sensitivity function can be depressed in patients with retinitis pigmentosa even in those who still have good central vision. ${ }^{2-7}$ Under constant luminance levels, the results of Arden grating tests showed that patients with retinitis pigmentosa have a decrease in their contrast sensitivity function, especially at high spatial frequencies. ${ }^{5-7}$ Measurements of contrast sensitivity using a modified Vistech contrast sensitivity system have shown that patients with retinitis pigmentosa have significantly lower contrast sensitivity at all luminance levels, especially at the middle and high spatial frequencies. ${ }^{7}$ Other authors have obtained similar results by using letter charts instead of grating targets. ${ }^{8-10}$ Their results showed that patients with retinitis pigmentosa had a lower ability to identify low contrast letters at all luminance levels. This contrast sensitivity method with letter targets has two advantages; first, the letters are easier to identify by patients, and second, the ability to use different luminance levels is easier, although the change in background luminance level is performed by a slide projector, which is not especially accurate.

Although contrast sensitivity measurements have been recognized to be a more sensitive method for following patients with retinitis pigmentosa, there are several difficulties that need to be overcome for its routine use in the outpatient clinic. First, most of the contrast sensitivity measurements are performed with printed charts which are difficult to print with accurate contrast. Second, patients can remember the orientation of the gratings or the position of the letter on a printed chart. And third, accurate and consistent luminance changes are not fully achieved with slide projectors.

The contrast sensitivity acuity tester (CAT-CP, Neits Instrument, Nagoya, Japan) was developed to overcome these disadvantages. This instrument determines the logarithm of the minimal angle of resolution (logMAR) visual acuity using different contrast Landolt rings instead of letter optotypes, and the tests can be performed under photopic and mesopic conditions. To date, the contrast visual acuity has been used mainly to assess preoperative and postoperative visual acuities in patients with cataracts, intraocular lens implantation, ${ }^{11}$ and refractive surgery. Contrast visual acuity findings in patients with retinitis pigmentosa have not been published.

The purpose of this study was to determine contrast visual acuity in patients with retinitis pigmentosa and compare this with that of normal individuals. We would like to confirm that measurements with lower contrast targets might be more representative of visual stimuli encountered in daily living by patients with retinitis pigmentosa.

\section{Materials and methods Subjects}

Retinitis pigmentosa was diagnosed on the findings of peripheral visual field restrictions and scotomas, abnormalities in the electroretinogram, intraretinal bone spicule-like pigmentation, and narrowing of the retinal vessels. Sixty eyes from 31 normal controls, 92 eyes from 52 typical patients with retinitis pigmentosa without cataract or other ophthalmological disorders (RP-1 group), and 20 eyes from 14 patients with typical retinitis pigmentosa and cataract without other ophthalmological disorders (RP-2 group) were studied. Cataract is known to influence contrast visual acuity even when visual acuity is apparently good, so we enrolled patients with retinitis pigmentosa and cataract as a different group (RP-2). The RP-2 enrolment criteria were cataract being cortical and posterior subcapsular type. The patients were seen in the Eye Clinic at Chiba University Hospital from May to November 2008. Their mean age ( \pm standard deviation) was $37.8 \pm 13.4$ (range $21-59$ ) years in the control group, $46.9 \pm 14.1$ (range 12 to 71 ) years in the RP-1 group, and $53.7 \pm 12.2$ (range 28-69) years in the RP-2 group. Only patients with retinitis pigmentosa whose decimal visual acuity was $\geq 1.0$ or $0 \log$ MAR units with central visual fields $\geq 10$ degrees were studied. Patients who had intraocular lens implantation, whose subjective refractive error was $> \pm 6.0$ diopters (D), or whose cylinder power was $> \pm 2.0 \mathrm{D}$ were excluded. Individuals with visual acuity $\geq 1.0$ and no ocular diseases were studied as controls.

\section{Conventional visual acuity measurements}

Visual acuity was determined monocularly using Landolt ring charts (system charts SC-2000 Nidek Instruments, Gamagori, Japan) at a test distance of $5 \mathrm{~m}$. All tests were performed at a constant background luminance of $150 \mathrm{~cd} / \mathrm{m}^{2}$. The decimal visual acuity values were converted to $\log$ MAR units, and mean values were used for the analyses. When more than two correct answers were obtained during the three trial tests, the subject was considered to have identified orientation of the ring correctly.

\section{Contrast visual acuity measurements}

Contrast visual acuity can be measured automatically under three contrast levels, ie, $100 \%, 10 \%$, and $5 \%$, under both 
photopic and mesopic conditions with the CAT-CP. However, only contrast levels of $100 \%$ and $10 \%$ were used, because most patients with retinitis pigmentosa had difficulty in recognizing even the largest ring of $1.3 \log$ MAR units under $5 \%$ contrast. Thus, contrast visual acuity was determined monocularly under four conditions, ie, two contrast levels under photopic and mesopic conditions. Landolt rings are used with the CAT-CP, and the luminance of the rings and background can be chosen to be photopic $\left(200 \mathrm{~cd} / \mathrm{m}^{2}\right)$ or mesopic $\left(10 \mathrm{~cd} / \mathrm{m}^{2}\right)$. The luminances of $200 \mathrm{~cd} / \mathrm{m}^{2}$ and $10 \mathrm{~cd} / \mathrm{m}^{2}$ were the conditions for daytime and evening, respectively. Measurements were made after dark-adapting for at least 5 minutes, and the refractive error was corrected for $5 \mathrm{~m}$. All measurements were made by one investigator (KO) who was masked to the type of patient.

\section{Comparisons}

Three comparisons were made. First, contrast visual acuities among the three groups were compared under the two contrast levels, $100 \%$ and 10\%, and under photopic and mesopic conditions. Second, differences between visual acuity $\left(150 \mathrm{~cd} / \mathrm{m}^{2}\right)$ and $100 \%$ contrast visual acuity under two luminance levels $\left(200 \mathrm{~cd} / \mathrm{m}^{2}\right.$ and $\left.10 \mathrm{~cd} / \mathrm{m}^{2}\right)$ were compared among the three groups to investigate the effects of luminance. In this comparison, we used the same Landolt ring under a $100 \%$ contrast level and chose the "distance" as $5 \mathrm{~m}$ on CAT-CP to minimize the difference between the two methods. Finally, the contrast visual acuities between two contrast levels of $100 \%$ and $10 \%$ among the three groups were compared under two luminance levels to investigate the effects of contrast.

\section{Statistical analysis}

The statistical significance of differences in visual acuity was determined using the analysis of covariance test (ANCOVA) for comparison among the three groups with correction for age differences. Differences in the contrast visual acuity data were also evaluated using ANCOVA with correction for age and visual acuity differences among the three groups.

ANCOVA is a general linear model with a continuous outcome variable (quantitative) and two or more predictor variables, where at least one is continuous (quantitative) and at least one is categorical (qualitative). Inclusion of covariates can increase the statistical power because it accounts for some of the variability. All of the probabilities were corrected for multiple testing by the Dunnett method. Differences were considered statistically significant if the $P$ value was $<0.05$.

\section{Results}

The visual acuities and contrast visual acuities of the three groups are shown in Table 1. The visual acuities were relatively good in the three groups, and were not significantly different by ANCOVA.

The $100 \%$ and $10 \%$ contrast visual acuities determined under photopic conditions were significantly lower in both the RP-1 and RP-2 groups than in the controls $(P=0.0001$, $P=0.0003, P=0.0002, P=0.0007$, respectively, by ANCOVA, Figure 1A). The $100 \%$ and $10 \%$ contrast visual acuities obtained under photopic conditions were significantly lower in the RP-2 group than in the RP-1 group ( $P=0.002, P=0.046$, respectively, by ANCOVA). The $100 \%$ and $10 \%$ contrast visual acuities determined under mesopic conditions were significantly lower in both the RP-1 and RP-2 groups than in the controls $(P=0.0002$, $P=0.0005, P=0.0009, P=0.0003$, respectively, by ANCOVA, Figure 1B). The $100 \%$ and $10 \%$ contrast visual acuities obtained under mesopic conditions were significantly lower in the RP-2 group than in the RP-1 group $(P=0.031$, $P=0.027$, respectively, by ANCOVA).

The differences between the visual acuities and the $100 \%$ contrast visual acuities under both photopic and mesopic conditions were significantly greater in both the RP-1 and RP-2 groups than in the controls $(P=0.0001$, $P=0.0004, P=0.0001, P=0.0003$, respectively, by ANCOVA, Figure 2A and B). Furthermore, the differences were significantly greater in the RP-2 group than in the RP-1 group under both photopic and mesopic conditions ( $P=0.048, P=0.047$, respectively, by ANCOVA, Figure 2A and $\mathrm{B})$. The differences between $100 \%$ contrast visual acuity and $10 \%$ contrast visual acuity under photopic and mesopic conditions were not significantly different between the three groups (by ANCOVA, Figure 3A and B).

\section{Discussion}

The $100 \%$ and $10 \%$ contrast visual acuities were significantly lower in the RP-1 and RP-2 groups than in the controls, even though all patients had good visual acuities. This is in good agreement with earlier findings. ${ }^{12}$ In addition, among patients

Table I Visual acuity and contrast visual acuity of the three groups

\begin{tabular}{lrrr}
\hline & \multicolumn{1}{l}{ Control } & \multicolumn{1}{l}{ RP-I } & \multicolumn{1}{c}{ RP-2 } \\
\hline Conventional VA & $-0.04 \pm 0.05$ & $-0.04 \pm 0.04$ & $-0.02 \pm 0.05$ \\
I00\% CVA, photopic & $0.00 \pm 0.05$ & $0.16 \pm 0.08$ & $0.28 \pm 0.09$ \\
I00\% CVA, mesopic & $0.14 \pm 0.06$ & $0.26 \pm 0.07$ & $0.4 \pm 0.09$ \\
I0\% CVA, photopic & $0.31 \pm 0.05$ & $0.47 \pm 0.07$ & $0.61 \pm 0.09$ \\
10\% CVA, mesopic & $0.53 \pm 0.06$ & $0.7 \pm 0.08$ & $0.84 \pm 0.09$ \\
\hline
\end{tabular}

Note: Data are expressed as the mean \pm standard deviation. 

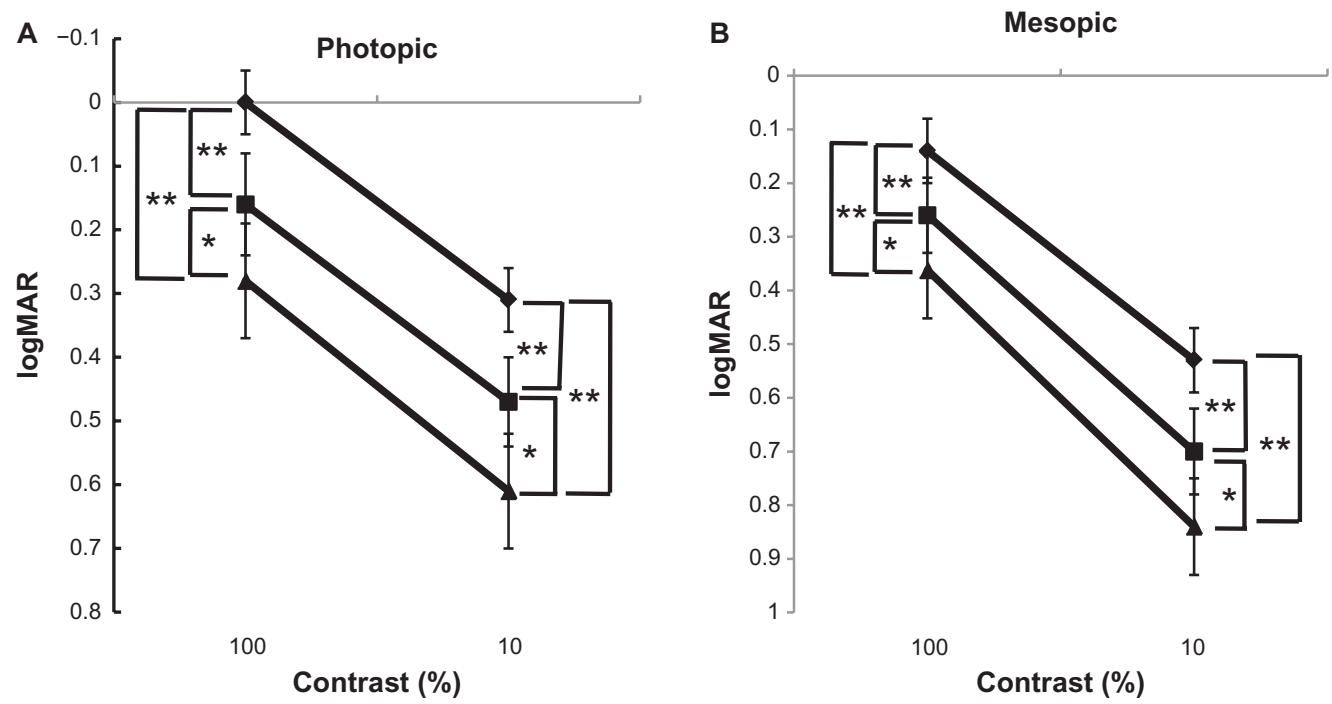

Figure I Measurement of contrast visual acuity. (A) Data obtained with $100 \%$ and 10\% contrast under photopic conditions. (B) Data obtained with $100 \%$ and I0\% contrast under mesopic conditions. control, $\square$ RP-I group, and $\Delta$ RP-2 group.

Notes: Data are the mean \pm standard deviation of three measurements. $* P<0.05$ and $* * P<0.001$.

with comparable visual acuities, patients with cataract had lower contrast visual acuity than those without cataract. This is supported by an earlier report that contrast visual acuity was more affected than the conventional visual acuity in patients with cataract. ${ }^{13}$

We also found among the patients with retinitis pigmentosa, but not in controls, that there were differences between visual acuity and $100 \%$ contrast visual acuity under photopic and mesopic conditions. The difference between visual acuity and $100 \%$ contrast visual acuity in the RP-1 and RP-2 groups was significantly greater than in controls, not only under mesopic but also under photopic background luminance. These findings indicate that there is a significant luminance effect on visual acuity. Considering that the visual acuities of both the RP-1 and RP-2 groups were good, a dysfunction of the rod may have affected the contrast visual acuity under the mesopic condition. Furthermore, the functional luminance range could be smaller in patients with retinitis pigmentosa, because the contrast visual acuity was also reduced under a photopic background, where the luminance was much higher than that of conventional visual acuity. There are several possibilities to explain this somewhat unexpected result. First, glaring under high luminance level affects visual acuity. In fact, we undertook a brief questionnaire survey of the participants, and found that they had visual difficulties from glaring, not only in the clinical evaluation but also in daily living when in a high luminance environment. Second, a different viewing method could have contributed to the difference. Visual acuity measurement uses an "open field view" chart, whereas contrast visual acuity with the Neitz CAT-CP uses a "closed field view". We tried to adjust for this difference by using the same Landolt ring on the same $100 \%$ contrast,
A

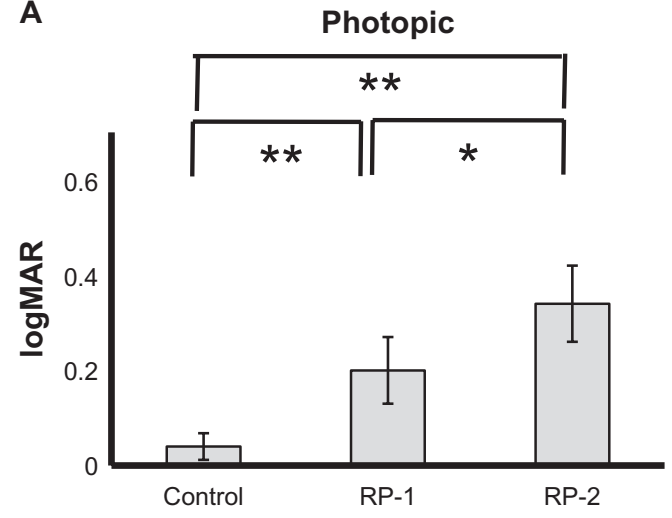

B

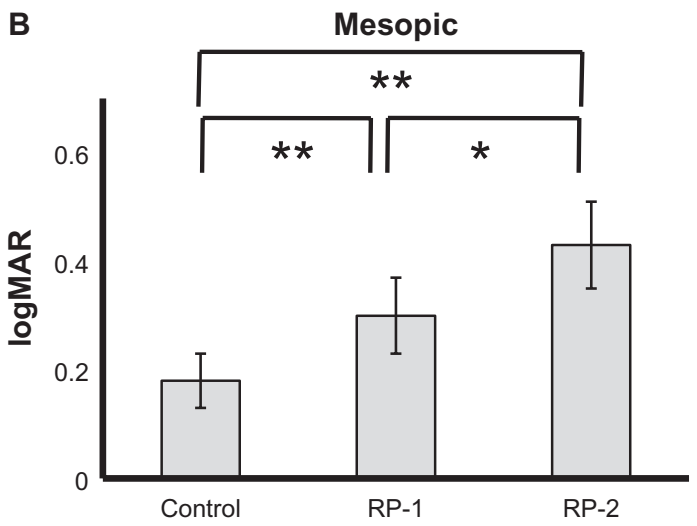

Figure 2 Differences between visual acuity and contrast visual acuity with $100 \%$ contrast under photopic (A) and mesopic conditions (B). Notes: Data represent the mean \pm standard deviation for three measurements. $* P<0.05$ and $* * P<0.001$. 
A

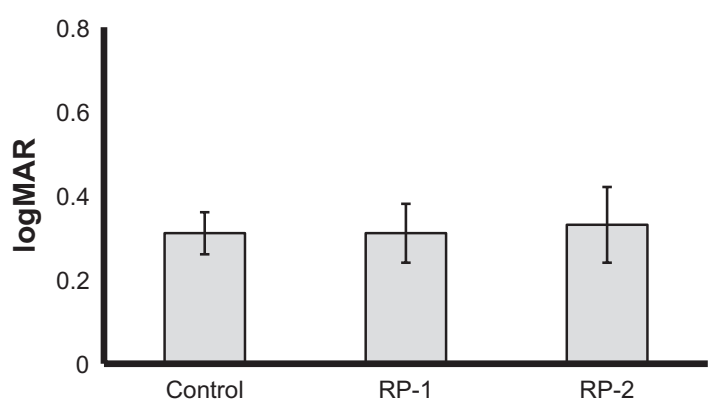

B Mesopic

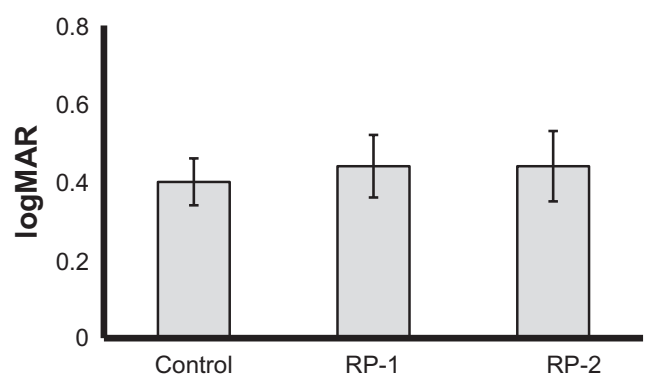

Figure 3 Differences in contrast visual acuity for 100\% and 10\% contrast under photopic (A) and mesopic (B) conditions. Note: Data are the mean \pm standard deviation of three measurements.

with the "distance" in CAT-CP as "long". In addition to that, we have adjusted the result statistically by using ANCOVA analysis. Thus, we believe that comparison of the two methods would be feasible. However, in a closed field view, in which the light source could be more directional, enhanced glaring and/or scattering might have a greater influence in patients with retinitis pigmentosa. If glaring really matters, tinted eye glasses may be helpful for patients with retinitis pigmentosa who have decreased photopic contrast visual acuity.

The difference between the $100 \%$ contrast visual acuity and the $10 \%$ contrast visual acuity under the same luminance was approximately the same among the three groups. These results indicate that reduction of target contrast affects visual acuity almost equally in normals and in patients with retinitis pigmentosa, even though the light response deteriorates significantly in patients with retinitis pigmentosa, which is apparent by the fact that patients could not recognize a $5 \%$ contrast Landolt ring.

In conclusion, our patients with retinitis pigmentosa and good visual acuity had significantly poorer contrast visual acuity. Therefore, when patients with retinitis pigmentosa and good visual acuity have subjective visual complaints, contrast visual acuity could be a sensitive method to monitor for subtle changes in foveal function.

\section{Acknowledgment}

The authors thank Emi Utsuno and Yasunori Sato for their contributions as study coordinators, and Duco Hamasaki for editing the manuscript.

Clinical Ophthalmology

\section{Publish your work in this journal}

Clinical Ophthalmology is an international, peer-reviewed journal covering all subspecialties within ophthalmology. Key topics include: Optometry; Visual science; Pharmacology and drug therapy in eye diseases; Basic Sciences; Primary and Secondary eye care; Patient Safety and Quality of Care Improvements. This journal is indexed on

\section{Disclosure}

The authors report no conflicts of interest in this work.

\section{References}

1. Farrar GJ, Kenna PF, Humphries P. On the genetics of retinitis pigmentosa and on mutation-independent approaches to therapeutic intervention. EMBO J. 2002;21(5):857-864.

2. Arden GB. The importance of measuring contrast sensitivity in cases of visual disturbance. Br J Ophthalmol. 1978;62(4):198-209.

3. Wolkstein M, Atkin A, Bodis-Wollner I. Contrast sensitivity in retinal disease. Ophthalmology. 1980;87(11):1140-1149.

4. Lindberg CR, Fishman GA, Anderson RJ, Vasquez V. Contrast sensitivity in retinitis pigmentosa. Br J Ophthalmol. 1981;65(12):855-858.

5. Marmor MF. Contrast sensitivity and retinal diseases. Ann Ophthalmol. 1981;13(9):1069-1071.

6. Marmor MF. Contrast sensitivity versus visual acuity in retinal disease. Br J Ophthalmol. 1986;70(7):553-559.

7. Sucs FE, Uvijls A. Contrast sensitivity in retinitis pigmentosa at different luminance levels. Clinical Vision Science. 1992;7(2):147-151.

8. Regan D. Do letter charts measure contrast sensitivity? Clinical Vision Science. 1991;6(5):401-408.

9. Alexander KR, Derlacki DJ, Fishman GA. Visual acuity vs letter contrast sensitivity in retinitis pigmentosa. Vision Res. 1995;35(10): $1495-1499$.

10. Spellman DC, Alexander KR, Fishman GA, Derlacki DJ. Letter contrast sensitivity in patients with retinitis pigmentosa assessed by Regan charts. Retina. 1989;9(4):287-291.

11. Hayashi K, Yoshida M, Hayashi H. All-distance visual acuity and contrast visual acuity in eyes with a refractive multifocal intraocular lens with minimal added power. Ophthalmology. 2009;116(3):401-408.

12. Akeo K, Hiida Y, Saga M, Inoue R, Oguchi Y. Correlation between contrast sensitivity and visual acuity in retinitis pigmentosa patients. Ophthalmologica. 2002;216(3):185-191.

13. Elliott DB. Evaluating visual function in cataract. Optom Vis Sci. 1993; 70(11):896-902.

\section{Dovepress}

PubMed Central and CAS, and is the official journal of The Society of Clinical Ophthalmology (SCO). The manuscript management system is completely online and includes a very quick and fair peer-review system, which is all easy to use. Visit http://www.dovepress.com/ testimonials.php to read real quotes from published authors. 